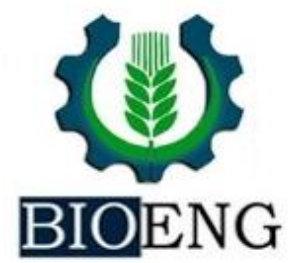

\title{
ESTUDO COMPARATIVO DO MÉTODO PADRÃO DA ESTUFA E DO MÉTODO SPEEDY NA DETERMINAÇÃO DO TEOR DE ÁGUA NO SOLO
}

${ }^{1}$ Centro Universitário de Lavras, Lavras, MG, Brasil.

${ }^{2}$ Centro Universitário de Formiga, Formiga, MG, Brasil.

${ }^{3}$ Universidade Federal de Lavras, Lavras, MG, Brasil.

${ }^{4}$ Universidade Federal Rural do Rio de Janeiro, Seropédica, Rio de Janeiro, Brasil.

Article history: Received 30 January 2018; Received in revised form 20 February 2018; Accepted 26 February 2018; Available online 27 March 2018.

\section{RESUMO}

A umidade do solo determina a quantidade de água retida nos espaços porosos do solo. $\mathrm{O}$ conhecimento desta propriedade é de suma importância, pois a água pode alterar o comportamento do solo. Dentre os métodos utilizados na avaliação dessa propriedade para avaliar essa propriedade destaca-se o método do speedy e o método da estufa. O objetivo desse trabalho foi comparar os resultados de umidade do solo obtidos através do método gravimétrico padrão de secamento em estufa e do método speedy. Foram coletadas amostras em triplicata de quatro classes de solo (Latossolo Vermelho, Latossolo Vermelho-Amarelo, Cambissolo e Argissolo), oriundas da cidade de Lavras - MG, provenientes da Universidade Federal de Lavras - UFLA, e analisadas em laboratório quanto a sua granulometria e umidade. Para determinar a umidade das amostras foram seguidos os procedimentos referentes as normas NBR 6457 para o método da estufa e as normas do Departamento Nacional de Estradas de Rodagem (DNER-ME 052/94) para o método do speedy. Foi possível verificar que o método do speedy forneceu valores de umidade abaixo daqueles determinados pelo método da estufa, possivelmente em virtude da dificuldade de desagregação total das partículas no interior do aparelho, de modo que, quanto maior a umidade do solo, mais os valores obtidos pelo método do speedy se afastaram daqueles obtidos pelo método da estufa. Observando-se as divergências encontradas, análises de regressão linear foram realizadas para obter uma equação de calibração do método do speedy para se atingir um valor mais aproximado do método da estufa. O modelo que melhor se ajustou foi $\mathrm{h}_{\mathrm{SC}}=46,292254-$ $333,088487 / \mathrm{h}_{\mathrm{SD}}$, apresentando um $\mathrm{R}^{2}=0,8843$.

Palavras-chave: água no solo, granulometria, umidade do solo com base em peso.

\section{COMPARATIVE STUDY OF THE STANDARD OVEN-DRYING AND THE SPEEDY METHOD FOR DETERMINING SOIL WATER CONTENT}

\begin{abstract}
Soil moisture determines the water content retained in the soil porous spaces. Knowledge of this property is essential, as water interfere on soil behavior. Among several methods available to evaluate this property, there is the speedy and oven-drying methods. The aim of this research was to compare soil moisture results obtained through the standard oven-drying method and the speedy method. Therefore, four soil class samples (Latossolo Vermelho,
\end{abstract}

\footnotetext{
* pedroterralima@yahoo.com.br
} 
Latossolo Vermelho-Amarelo, Cambissolo and Argissolo) were collected in triplicate, from Lavras - MG, Brazil, at Federal University of Lavras - UFLA, and analyzed for particle-size and moisture content. In order to quantify soil moisture, oven-drying method was performed according to NBR 6457 procedures, whereas speedy method was realized according to procedures from National Department of Highways (DNER-ME 052/94). It was verified that the speedy method provided values of soil moisture lower than those determined by the ovendrying method, possibly due to the difficulty of the particles total disintegration inside the apparatus, wherein the soil moisture increase led to higher differences between methodologies. According to the divergences stated between methods, linear regression analyzes were performed to obtain a calibration equation of the speedy method to reach a more approximate value in relation to the oven-drying method. The best fitting model was $\mathrm{h}_{\mathrm{SC}}=46,292254-333,088487 / \mathrm{h}_{\mathrm{SD}}$, with a $\mathrm{R}^{2}=0,8843$.

Keywords: water content, particle-size, soil moisture, gravimetric soil moisture.

\section{INTRODUÇÃO}

Para realizar o monitoramento da umidade do solo, existem diversas maneiras e técnicas que podem ser utilizadas, segundo métodos diretos ou indiretos, cada qual apresentando uma dada precisão, tempo de resposta e custo diferenciado em função dos equipamentos e recursos tecnológicos envolvidos (FONSECA et al., 2009).

Os métodos de determinação da umidade do solo estão classificados em diretos, como o gravimétrico, ou indiretos, como tensiômetros ou blocos de resistência elétrica. Os métodos diretos consistem na medida direta do conteúdo de água de uma amostra por meio de sua remoção, basicamente por evaporação. Enquanto que os métodos indiretos envolvem a medição de alguma propriedade física ou físicoquímica do solo que seja proporcional ao conteúdo de água. Desta forma, são métodos que exigem uma curva de calibração e apresentam as vantagens de serem não destrutivos e proporcionam menor tempo e trabalho na execução das medidas (VIONE, 2007).

$\mathrm{O}$ método gravimétrico (método da secagem em estufa) é o método direto considerado padrão. Nele, a massa de água presente numa dada amostra de solo é obtida pela diferença entre o peso da amostra úmida e seca (SILVA, 2013). Este método é adotado como padrão devido à sua simplicidade, praticidade e principalmente precisão, sendo utilizado como referência para calibrar equipamentos e métodos (BUSKE et al., 2013).

Por outro lado, apresenta desvantagens por ser um método destrutivo e demorado, impossibilitando a obtenção de resultados instantaneamente (SILVA, 2013). Outra desvantagem é o tempo necessário para a obtenção do resultado, que é de aproximadamente 24 horas. Porém, este método é um dos mais utilizados pelo seu baixo custo de realização, não necessitando de equipamentos sofisticados e nem de adição de produtos químicos como outros métodos diretos (BUSKE et al., 2013).

Caracterizado como um método indireto, o método speedy é normalizado pelo Departamento Nacional de Estradas e Rodagem - DNER-ME 052/94. Neste método utiliza-se o conjunto speedy, que é um aparelho constituído por um reservatório metálico fechado responsável por fazer a comunicação com um manômetro destinado a medir a pressão interna. Dentro deste reservatório, são colocadas duas esferas de aços que, por agitação manual, entrarão em contato com uma quantidade de solo úmido e carbureto de cálcio $\left(\mathrm{CaC}_{2}\right)$ (BRAGA et al., 2009). A água contida no solo, em combinação com o $\mathrm{CaC}_{2}$, gera acetileno $\left(\mathrm{C}_{2} \mathrm{H}_{2}\right)$ e hidróxido de cálcio e a partir da variação da pressão interna obtém-se a quantidade de água existente no solo (CAPUTO, 2015). 
Quando o manômetro registrar pressão interna constante, encerra-se o ensaio. De posse da leitura manométrica e do peso da amostra utilizados nos ensaios, obtém-se o valor da umidade do solo através de uma tabela de aferição, própria do aparelho (DNER-ME 052/94).

Esse método de speedy caracteriza-se pela facilidade de manuseio dos equipamentos, adequabilidade a qualquer solo e qualquer faixa de umidade, porém, é

\section{MATERIAL E MÉTODOS}

O presente trabalho foi realizado no Centro Educacional de Lavras UNILAVRAS na cidade de Lavras - MG, onde foram verificadas as umidades, com base em peso, de amostras de 4 (quatro) classes de solos. Para definir as umidades das amostras dos solos foram utilizados dois métodos: o método padrão da estufa e o método expedito do speedy.

Realizou-se a amostragem das classes de solo avaliadas, sendo elas: Latossolo Vermelho (LV), Latossolo Vermelho-Amarelo (LVA), Cambissolo (CX) e Argissolo (PA), oriundas da cidade de Lavras - MG, provenientes da Universidade Federal de Lavras - UFLA.

Foram coletadas, em locais representativos escolhidos aleatoriamente, amostras com estruturas deformadas dos solos estudados, na camada superficial de 0 a $10 \mathrm{~cm}$. Para a coleta das amostras, foi removida a cobertura vegetal da camada superficial e, antes do acondicionamento das amostras, o excesso de raízes contido no solo coletado foi removido manualmente.

As amostras de solos com estruturas deformadas foram acondicionadas em sacos plásticos e coletadas em quantidades suficientes para a realização de ensaios de determinação da umidade e granulometria.

As amostras destinadas à avaliação da umidade dos solos foram encaminhadas ao Laboratório de Mecânica dos Solos no Centro Educacional de Lavras UNILAVRAS, onde foram secadas em estufa a $100^{\circ} \mathrm{C}$ durante 24 horas, para que posteriormente fossem adicionadas necessária calibragem. A determinação da umidade ocorre em poucos minutos e o custo é relativamente baixo. Todavia, tratase de um método destrutivo (VIELMO, 2008).

Diante do exposto, o objetivo desse trabalho foi comparar os resultados de umidade do solo obtidos através do método gravimétrico padrão de secamento em estufa e do método speedy.

diferentes quantidades de água correspondentes aos teores de água para os diferentes tratamentos $(10,20$ e $30 \%)$. As amostras destinadas à determinação da textura dos solos foram encaminhadas ao Laboratório de Física dos Solos do Departamento de Ciência do Solo da Universidade Federal de Lavras - UFLA.

Para realização do ensaio do método da estufa, foram seguidos os procedimentos descritos na Norma Brasileira Regulamentadora - NBR 6457 da Associação Brasileira de Normas Técnicas - ABNT (1986). Para a realização do ensaio do método do speedy foram seguidos os procedimentos descritos na norma DNER-ME 052/94.

\section{Preparo das amostras}

As amostras deformadas secas em estufa foram colocadas num almofariz e, com o auxílio de um pistilo, foram destorroadas. Após o destorroamento foi realizado um peneiramento do solo, utilizando uma peneira de 4,75 milímetros (ABNT, 1986).

Para cada classe de solo avaliada, a amostra de solo seca em estufa, destorroada e peneirada, foi dividida em três porções de $250 \mathrm{~g}$ cada, que foram colocadas numa bandeja metálica. Em seguida, calculou-se a quantidade de água a ser adicionada a cada porção de solo seco para compor as amostras que seriam submetidas aos ensaios de determinação de umidade.

Neste trabalho, optou-se por avaliar amostras com umidade de 10\%, $20 \%$ e 
$30 \%$, em peso, pois correspondem às faixas de valores de umidade comumente encontradas em campo. Uma vez que a umidade gravimétrica do solo é definida como a relação entre a massa de água e a massa de sólidos de uma amostra de solo, a massa de água a ser adicionada a cada porção de $250 \mathrm{~g}$ de sólidos (solo seco) para obtenção das amostras com teores de umidade iguais a $10 \%, 20 \%$ e $30 \%$ foi realizada a partir da Equação 1.

$$
\mathrm{M}_{\text {água }}=\left(\mathrm{u} \text { x } \mathrm{M}_{\text {sólidos }}\right) / 100
$$

\section{Em que:}

$\mathrm{M}_{\text {água }}$ - massa de água a ser adicionada aos sólidos, em gramas $(\mathrm{g})$.

$\mathrm{u}$ - umidade desejada para a amostra de solo, em porcentagem (\%).

$\mathrm{M}_{\text {sólidos - massa de sólidos (solo seco) que }}$ irá compor a amostra, em gramas (g).

Com o auxílio de um becker e uma seringa plástica de $10,0 \mathrm{ml}$, a quantidade de água calculada pela Equação 1 foi pesada em uma balança de precisão.

A quantidade de água calculada para elevar a porção de solo seco às umidades de $10 \%, 20 \%$ e $30 \%$ foi adicionada às bandejas contendo as porções de solo seco, homogeneizando-se a mistura. Assim, cada bandeja recebeu uma quantidade de água diferente, obtendo-se, para cada classe de solo avaliada, três amostras a serem ensaiadas: uma com $10 \%$, outra com $20 \%$ e outra com $30 \%$ de umidade gravimétrica.

Cada amostra de solo preparada, representativa de cada umidade desejada, foi subdividida em 3 (três) sub-amostras que tiveram suas umidades determinadas pelo método da estuda e pelo método do speedy.

\section{Determinação da umidade pelo método padrão da estufa}

De acordo com a NBR 6457 (ABNT, 1986), a umidade gravimétrica do solo é obtida através da Equação 2 apresentada a seguir.

$$
\mathrm{h}=[(\mathrm{M} 1-\mathrm{M} 2) /(\mathrm{M} 2-\mathrm{M} 3)] \times 100
$$

Em que:

$\mathrm{h}$ - umidade do solo, expresso em porcentagem $(\%)$.

M1 - massa do solo úmido mais a massa do recipiente, expresso em gramas $(\mathrm{g})$.

M2 - massa do solo seco mais a massa do recipiente, expresso em gramas $(\mathrm{g})$.

M3 - massa do recipiente, expresso em gramas $(\mathrm{g})$.

\section{Determinação da umidade pelo método expedito do speedy}

Primeiramente, determinou-se a quantidade de amostra úmida a ser adicionada à câmara do conjunto speedy, conforme Tabela 1. Assim, os ensaios foram realizados através do uso de 20 gramas das amostras preparadas para ficarem com $10 \%$ de umidade, 5 gramas das amostras preparadas para ficarem com $20 \%$ de umidade; e 3 gramas das amostras preparadas para ficarem com $30 \%$ de umidade.

Tabela 1. Peso da amostra em função da umidade gravimétrica admitida

\begin{tabular}{cc}
\hline $\begin{array}{c}\text { Umidade estimada } \\
(\%)\end{array}$ & $\begin{array}{c}\text { Peso da amostra } \\
(\mathrm{g})\end{array}$ \\
\hline 5 & 20 \\
10 & 10 \\
20 & 5 \\
30 ou mais & 3 \\
\hline
\end{tabular}

Posteriormente, foram introduzidas na câmara do aparelho a quantidade de amostra úmida pré-estabelecida, duas esferas de aço e uma a ampola de carbureto de cálcio, sendo que este último, após agitação manual, reagiu com a água e gerou o gás acetileno. A pressão gerada pelo gás foi verificada no manômetro.

Por fim, a pressão manométrica foi lida após se apresentar constante, o que 
indica que toda a água existente na amostra reagiu com o carbureto.

Com base no resultado da pressão assinalada no manômetro, no peso da amostra inserida na câmara do aparelho e na tabela de aferição própria do aparelho, obteve-se a porcentagem de umidade em relação à amostra total úmida, por meio da Tabela 2.

Para determinar a umidade gravimétrica em relação ao peso do solo seco, foi utilizada a Equação 3 de acordo com o DNER-ME 052/94.

$\mathrm{h}=[\mathrm{h} 1 /(100-\mathrm{h} 1)] \times 100$

Em que:

h - teor de umidade em relação ao solo seco, expresso em porcentagem (\%).

h1 - umidade dada pelo aparelho speedy em relação à amostra total úmida, expresso em porcentagem $(\%)$.

Tabela 2. Valores obtidos para Pressão x Umidade

\begin{tabular}{ccccc}
\hline \multirow{2}{*}{$\begin{array}{c}\text { Leitura no } \\
\text { manômetro }\end{array}\left(\mathrm{kg} / \mathrm{cm}^{2}\right)$} & \multicolumn{4}{c}{ \% de umidade em função do peso } \\
\cline { 2 - 5 } & $20 \mathrm{~g}$ & $15 \mathrm{~g}$ & $10 \mathrm{~g}$ & $5 \mathrm{~g}$ \\
\hline 0,10 & 0,3 & 1,3 & 1,5 & 2,5 \\
0,15 & 0,6 & 1,8 & 2,2 & 3,5 \\
0,20 & 1,0 & 2,2 & 2,8 & 4,4 \\
0,25 & 1,3 & 2,7 & 3,4 & 5,3 \\
0,30 & 1,7 & 3,2 & 4,0 & 6,3 \\
0,35 & 2,1 & 3,6 & 4,7 & 7,2 \\
0,40 & 2,5 & 4,1 & 5,3 & 8,2 \\
0,45 & 2,9 & 4,6 & 5,9 & 9,1 \\
0,50 & 3,3 & 5,1 & 6,5 & 10,1 \\
0,55 & 3,7 & 5,6 & 7,2 & 11,0 \\
0,60 & 4,1 & 6,1 & 7,7 & 11,9 \\
0,65 & 4,5 & 6,6 & 8,3 & 12,9 \\
0,70 & 4,9 & 7,0 & 8,9 & 13,8 \\
0,75 & 5,3 & 7,5 & 9,6 & 14,8 \\
0,80 & 5,7 & 8,0 & 10,2 & 15,7 \\
0,85 & 6,1 & 8,5 & 10,8 & 16,7 \\
0,90 & 6,5 & 8,9 & 11,4 & 17,6 \\
0,95 & 6,9 & 9,4 & 12,1 & 18,5 \\
1,00 & 7,3 & 9,9 & 12,8 & 19,5 \\
1,05 & 7,7 & 10,4 & 13,4 & 20,5 \\
1,10 & 8,1 & 10,9 & 14,0 & 21,5 \\
1,15 & 8,5 & 11,4 & 14,6 & 22,4 \\
1,20 & 8,9 & 11,9 & 15,2 & 23,5 \\
1,25 & 9,3 & 12,3 & 15,9 & 24,5 \\
\hline & & & &
\end{tabular}




\begin{tabular}{ccccc}
\hline 1,30 & 9,7 & 12,8 & 16,4 & 25,4 \\
1,35 & 10,1 & 13,3 & 17,1 & 26,4 \\
1,40 & 10,5 & 13,8 & 17,7 & 27,3 \\
1,45 & 10,9 & 14,2 & 18,3 & 28,4 \\
1,50 & 11,3 & 15,7 & 19,9 & 29,3 \\
\hline
\end{tabular}

Foi possível observar que na tabela 2 de aferição do conjunto speedy utilizado nos ensaios não apresenta os valores de calibração para ensaios realizados utilizando-se 3,0 g de amostra úmida.

A umidade dada pelo aparelho Speedy, para as amostras de $30 \%$ de umidade, foi ajustada pela Equação 4, apresentada a seguir, de acordo com os dados obtidos na Tabela 2. O ajuste da Equação 4 apresentou um coeficiente de determinação $\left(\mathrm{R}^{2}\right)$ igual a 0,9943 e permitiu estimar os valores de h1 para os ensaios que foram realizados com $3 \mathrm{~g}$ de amostra úmida.

$\mathrm{h} 1=56,05 \cdot \mathrm{P}^{-0,655} \cdot \mathrm{L}^{\left(0,0027 \cdot \mathrm{P}^{2}-0,0495 \cdot \mathrm{P}+1,1285\right)}$

Em que:

h1 - umidade dada pelo aparelho Speedy em relação à amostra total úmida, expresso em porcentagem $(\%)$.

\section{RESULTADOS E DISCUSSÃO}

Os resultados da análise granulométrica e a classificação textural
$\mathrm{P}$ - peso da amostra úmida utilizada no ensaio, em gramas ( $\mathrm{g})$.

L - leitura no manômetro, em quilograma por centímetro quadrado $\left(\mathrm{kg} / \mathrm{cm}^{2}\right)$.

\section{Análise estatística dos dados}

Os dados de umidade obtidos foram submetidos a análises de variância, sendo aplicado o Teste de Scott - Knott para avaliação das médias, a um nível de significância de $5 \%$.

Ao utilizar os valores médios dos parâmetros determinados para cada solo estudado, foram realizadas análises de regressão linear simples entre os métodos de determinação de umidade avaliados.

As análises estatísticas foram realizadas utilizando-se o software Sisvar (FERREIRA, 2008).

dos solos estudados estão representados na Tabela 3. 
Tabela 3. Granulometria e classificação textural dos solos estudados.

\begin{tabular}{|c|c|c|c|c|}
\hline \multirow{3}{*}{ Solo } & \multicolumn{3}{|c|}{ Granulometria } & \multirow{3}{*}{$\begin{array}{c}\text { Classe } \\
\text { textural }^{(1)}\end{array}$} \\
\hline & Areia & Silte & Argila & \\
\hline & \multicolumn{3}{|c|}{$\%$} & \\
\hline LV & 27 & 17 & 56 & $\begin{array}{c}\text { Argila } \\
\text { areno-siltosa }\end{array}$ \\
\hline LVA & 57 & 8 & 35 & $\begin{array}{c}\text { Areia } \\
\text { argilosa }\end{array}$ \\
\hline $\mathrm{CX}$ & 54 & 12 & 34 & $\begin{array}{l}\text { Areia argilo- } \\
\quad \text { siltosa }\end{array}$ \\
\hline PA & 50 & 14 & 36 & $\begin{array}{l}\text { Areia argilo- } \\
\text { siltosa }\end{array}$ \\
\hline
\end{tabular}

Houve a predominância da fração areia nas amostras de solos analisadas, com exceção do LV. Os Latossolos Vermelhos são solos de baixos conteúdos de silte, alto grau de intemperismo e sua textura pode variar desde média até muito argilosa (SANTOS et al., 2013).

As amostras de solo analisadas representam a camada superficial (0-10 $\mathrm{cm})$ e, a esse respeito, os Argissolos (PA) apresentam acúmulo de argila em profundidade, devido à mobilização e perda de argila da parte mais superficial do solo (EMBRAPA, 2016a) e muitos Latossolos Vermelho-Amarelos (LVA) podem apresentar comportamento argissólico (EMBRAPA, 2016b). Já os Cambissolos $(\mathrm{CX})$ possuem características bastante variáveis, incluindo à textura, e são reconhecidos pelo baixo grau de desenvolvimento pedogenético (SANTOS et al., 2013).

A Tabela 4 apresenta os valores médios de umidade determinados para os solos estudados. Comparando-se os valores obtidos pelo método da estufa com os obtidos pelo método do speedy, observa-se que os valores não diferiram estatisticamente apenas para as amostras com baixa umidade (10\%). Para umidades maiores (20\% e $30 \%$ ), os valores obtidos pelo método speedy foram menores que os obtidos pelo método da estufa, para todos os solos analisados.

Braga et al. (2009), ao compararem diferentes métodos de determinação do teor de umidade do solo, também verificaram diferenças significativas entre o método do speedy e o método da estufa. Os autores observaram que naquelas umidades em torno de $10 \%$ e $20 \%$, os resultados não foram satisfatórios, demonstrando que o speedy utilizado não estava devidamente calibrado.

Bragança et al. (2010); Feitosa et al. (2012) também observaram, em seus estudos, que as determinações de umidade dos solos realizadas com equipamento speedy sempre forneciam valores menores que a umidade real, dada pelo método da estufa.

Com relação à influência da textura na precisão do método do speedy, pode-se perceber que as variações granulométricas dos solos estudados, de um modo geral, não influenciaram nos resultados de umidade, demonstrando a eficácia das metodologias avaliadas. A esse respeito, Berney et al. (2011) compararam o método do speedy com o método da estufa para amostras de solos de textura argilosa, mediana e arenosa e verificaram que os coeficientes de correlação entre os valores encontrados não variaram em função da textura do solo. 
Tabela 4. Valores médios de umidade gravimétrica determinados pelo método padrão da estufa e pelo método expedito do speedy para os solos analisados.

\begin{tabular}{|c|c|c|c|c|c|}
\hline \multirow{2}{*}{ Tratamento } & \multirow{2}{*}{$\begin{array}{c}\text { Método de } \\
\text { determinação }\end{array}$} & \multicolumn{4}{|c|}{ Solo } \\
\hline & & $\mathbf{L V}$ & LVA & $\mathbf{C X}$ & PA \\
\hline Amostra & Estufa & $11,66 \mathrm{Aa}$ & $11,01 \mathrm{Aa}$ & $10,85 \mathrm{Aa}$ & $10,92 \mathrm{Aa}$ \\
\hline $\begin{array}{l}\text { preparada com } \\
10 \% \text { de umidade }\end{array}$ & Speedy & $10,05 \mathrm{Ba}$ & $9,29 \mathrm{Aa}$ & $10,34 \mathrm{Ba}$ & $10,34 \mathrm{Ba}$ \\
\hline Amostra & Estufa & $21,09 \mathrm{Ab}$ & $20,71 \mathrm{Ab}$ & $21,01 \mathrm{Ac}$ & $20,78 \mathrm{Ac}$ \\
\hline $\begin{array}{l}\text { preparada com } \\
20 \% \text { de umidade }\end{array}$ & Speedy & $13,25 \mathrm{Aa}$ & $13,94 \mathrm{Aa}$ & $16,88 \mathrm{Ab}$ & $15,21 \mathrm{Ab}$ \\
\hline Amostra & Estufa & $30,72 \mathrm{Ac}$ & $30,37 \mathrm{Ac}$ & $30,25 \mathrm{Ad}$ & $30,23 \mathrm{Ad}$ \\
\hline $\begin{array}{l}\text { preparada com } \\
30 \% \text { de umidade }\end{array}$ & Speedy & $16,49 \mathrm{Aa}$ & $20,36 \mathrm{Ab}$ & $16,99 \mathrm{Ab}$ & $15,77 \mathrm{Ab}$ \\
\hline
\end{tabular}

Médias seguidas pela mesma letra minúscula nas colunas e pela mesma letra maiúscula nas linhas não diferem entre si pelo teste de Scott-Knott, a 5\% de probabilidade.

A recomendação de Santos (2008) é que a aplicação do método speedy limite-se aos solos granulares, uma vez que nos solos finos, principalmente os argilosos, a precisão dos resultados são menores, em virtude da dificuldade de desagregação total das partículas no interior do aparelho.
Mas essa perda de precisão provocada pela variação de textura não foi constatada neste trabalho.

A Figura 1 permite confirmar a necessidade da calibração do aparelho speedy utilizado.

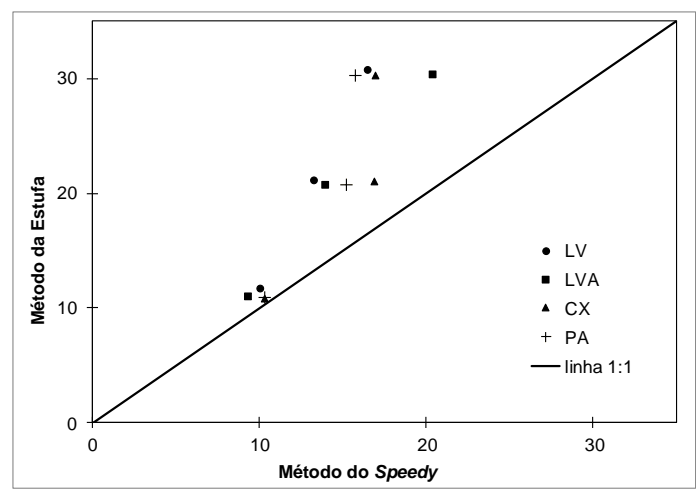

Figura 1. Umidade gravimétrica determinada pelo método expedito do speedy versus umidade gravimétrica determinada pelo método padrão da estufa. Nota: unidade de medida dos valores dos eixos coordenados: porcentagem.

Os valores determinados pelo método speedy ficaram próximos dos valores padrão determinados pelo método da estufa somente para as amostras de baixa umidade (10\%), podendo-se observar a pequena dispersão dos pontos em torno da linha 1:1. Esta linha indica o que seria um comportamento perfeito dos dados, ou seja, quanto mais próximos os pontos estiverem dela, maior a precisão das determinações indiretas de umidade.

Reforçando o que já foi exposto anteriormente, para as amostras de maior umidade (20\% e 30\%), os resultados do método do speedy não foram satisfatórios uma vez que os pontos se afastaram mais 
da linha 1:1, mostrando maior dispersão dos resultados.

Verifica-se também que, quanto maior a umidade da amostra, menor a precisão da determinação realizada pelo método do speedy (mais os pontos de afastam da linha 1:1).

Procurando identificar a relação entre os métodos de determinação de umidade do solo utilizados nesse trabalho, foram realizadas análises de regressão linear simples com o intuito de encontrar uma equação matemática que permitisse corrigir os valores encontrados pelo método do speedy para os solos analisados.

$\mathrm{O}$ modelo que melhor se ajustou aos dados (Equação 5) é apresentado a seguir.

$\mathrm{h}_{\mathrm{SC}}=46,292254-333,088487 / \mathrm{h}_{\mathrm{SD}}$

Em que:

$\mathrm{h}_{\mathrm{SC}}=$ umidade corrigida, em porcentagem .
$\mathrm{h}_{\mathrm{SD}}=$ umidade determinada pelo método do speedy, em porcentagem.

O ajuste da Equação 5 apresentou um coeficiente de determinação $\left(\mathrm{R}^{2}\right)$ igual a 0,8843 , o que indica que o modelo consegue explicar, satisfatoriamente, $88,43 \%$ dos dados analisados. Esta equação pode ser utilizada para a correção das umidades obtidas através do aparelho speedy utilizado neste estudo, pois reduz bastante à dispersão dos valores obtidos quando comparados àqueles determinados pelo método padrão da estufa, conforme mostra a Figura 2.

A equação proposta, portanto, pode ser considerada uma equação de calibração do equipamento speedy utilizado, permitindo-se chegar a valores de umidade mais precisos, com os valores preditos estando mais próximos da linha 1:1, proporcionando menores erros.

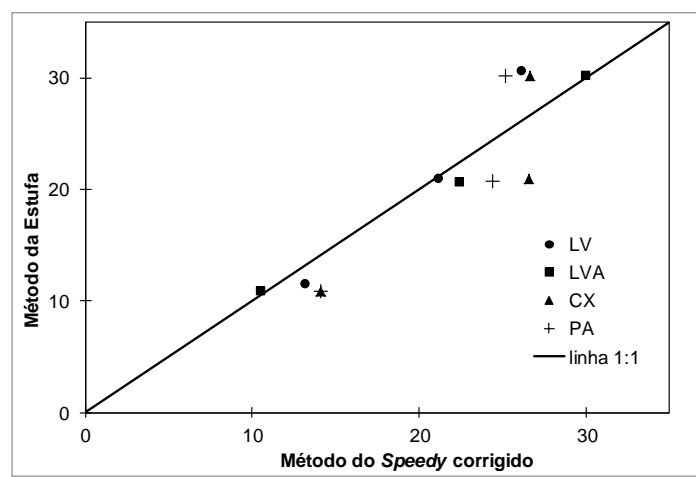

Figura 2. Umidade gravimétrica corrigida determinada pelo método expedito do speedy versus umidade gravimétrica determinada pelo método padrão da estufa. Nota: unidade de medida dos valores dos eixos coordenados: porcentagem.

$\mathrm{Na}$ engenharia civil, os estudos das propriedades dos solos são realizados geralmente em construções de grande porte, sendo que, para a determinação da umidade do solo, o método do speedy é mais utilizado pela sua praticidade e rapidez na obtenção dos resultados (SOTELO et al., 2014). A utilização deste

\section{CONCLUSÕES}

O método do speedy fornece valores de umidade gravimétrica abaixo daqueles determinados pelo método da estufa em método sem levar em consideração a precisão das determinações pode acarretar em resultados não tão eficazes quanto aos resultados provindos pelo método da estufa. Daí a necessidade dos procedimentos de calibração, a qual foi evidenciada nesse trabalho.

teores de umidade mais elevados $(20 \%$ e $30 \%)$.

A granulometria dos solos não interferiu significativamente nas 
determinações de umidade, em ambos os métodos analisados.

O método do speedy poderá ser confiável desde que seja devidamente calibrado.

\section{REFERÊNCIAS BIBLIOGRÁFICAS}

ASSOCIAÇÃO BRASILEIRA DE NORMAS TÉCNICAS. NBR 6457/86: Amostras de solo - Preparação para Ensaios de Compactação e Ensaios de Caracterização. Rio de Janeiro, 1986.

BERNEY, I. V.; ERNEST, S.; KYZAR, J. D.; OYELAMI, L. O. Device comparison for determining field soil moisture content (No. ERDC/GSL-TR-11-42). Engineer Research and Development Center Vicksburg MS Geotechnical and Structures Lab. 2011.

BRAGA, G. V. B dos P.; RIBEIRO, M. de J. S.; FERREIRA, P. M. M.; GUIMARÃES, R. O.; SANTOS, T. L. dos; VIANA, P. M. F. Comparação de métodos de determinação do teor de umidade. 2009. Disponível em: <http://www.prp2.ueg.br/sic2010/fronteira/ arquivos/trabalhos_2009/engenharias/jorna da/comparacao_de_metodos.pdf $>$. Acesso em 19/12/2017.

BRAGANÇA, R.; BRUM, V. J.; SILVA, J. M.; ZINI JÚNIOR, A.; BREGONCI, I. S.; REIS, E. F.; PASSOS, R. R. Avaliação da umidade de um latossolo da região sul do estado do espírito santo por diferentes métodos de determinação. IX Encontro Latino Americano de Iniciação Científica e V Encontro Latino Americano de PósGraduação - Universidade do Vale do Paraíba. 2010. Anais... Disponível em: <http://biblioteca.univap.br/dados/INIC/cd/ epg/epg5/epg5-10.pdf $>$. Acesso em 20/03/2016.

BUSKE, T. C.; ROBAINA, A. D.; PEITER, M. X.; TORRES, R. R.; ROSSO, R. B.; BRAGA, F. de V. A. Determinação da umidade do solo por diferentes fontes
A equação de correção ajustada permitiu reduzir a dispersão dos dados obtidos pelo método do speedy em relação aos dados obtidos pelo método da estufa.

de aquecimento.Irriga, Botucatu, v. 19, n. 2, p. 315-324, abril-junho, 2014.

CAPUTO, H. P. Mecânica dos solos e suas aplicações: fundamentos. 6. ed., v.1, São Paulo: LTC, 2015.

DEPARTAMENTO NACIONAL DE ESTRADAS E RODAGEM. DNER-ME 052: Solos e agregados miúdos Determinação da umidade com emprego do "Speedy". Rio de Janeiro, 4 p., 1994.

EMPRESA BRASILEIRA DE PESQUISA AGROPECUÁRIA - EMBRAPA. Argissolos. 2016a. Disponível em: <http://www.agencia.cnptia.embrapa.br/ge stor/solos_tropicais/arvore/CONTAG01_7 _2212200611538.html>. Acesso em 21/08/2017.

EMPRESA BRASILEIRA DE PESQUISA AGROPECUÁRIA - EMBRAPA. Latossolos Vermelho-Amarelos. 2016b. Disponível em: <http://www.agencia.cnptia.embrapa.br/ge stor/solos_tropicais/arvore/CONT000g05ip 3qr02wx5ok0q43a0r3t5vjo4.html $>$.

Acesso em 21/08/2017.

FEITOSA, J. R. L.; BANDEIRA, A. P. N.; CAVALCANTE, O. R. O. Importância da calibração dos equipamentos de leitura utilizados no laboratório de mecânica dos solos. IV Encontro Universitário da UFC no Cariri. Juazeiro do Norte: UFC, 2012. Anais... Disponível em: $<$ https://encontros.ufca.edu.br/index.php/e ncontros-universitarios/eu-

2012/paper/viewFile/1271/787>. Acesso em $20 / 01 / 2018$.

FERREIRA, D.F. SISVAR: Um programa para análises e ensino de estatística. 
Revista Symposium, Lavras, v. 6, p. 3641, 2008.

FONSECA, S. O., ARAÚJO, G. L., FARIA, B. H. G., LIPARIZI JUNIOR, A., COSTA, J., REIS, E. F. Avaliação do método do forno microondas para a determinação de umidade do solo em relação ao método padrão de estufa. XIII Encontro Latino Americano de Iniciação Científica e IX Encontro Latino Americano de Pós-Graduação - Universidade do Vale do Paraíba. 2009. Anais... Disponível em: <http://www.inicepg.univap.br/cd/INIC_2 009/anais/arquivos/RE_0213_0306_01.pdf >. Acesso em 19/12/2017.

SANTOS, J. A. Compactação: elementos teóricos. 2008. Disponível em: $<$ http://www.civil.ist.utl.pt/ jaime/Compac ta_T.pdf $>$. Acesso em 21/08/2017.

SANTOS, H. G.; JACOMINE, P. K. T.; ANJOS, L. H. C.; OLIVEIRA, V. A.; OLIVEIRA, J. B.; COELHO, M. R.; LUMBRERAS, J. F.; CUNHA, T. J. F. Sistema brasileiro de classificação de solos. 3. ed. Rio de Janeiro: Embrapa Solos; 2013.
SILVA, D. O. M. da. Validação de um sensor de determinação da umidade do solo para o manejo da irrigação. 2013. 63 f. Dissertação (Mestrado em Engenharia Agrícola), Universidade Federal do Vale do São Francisco, Juazeiro.

SOTELO, M.J.; MAZARI, M.; GARIBAY, J.; NAZARIAN, S. Variability of moisture content measurement devices on subgrade soils. Geo-Congress 2014: Geo-characterization and Modeling for Sustainability. Anais... Disponível em: <http:// https://ascelibrary.org/doi/pdf/10.1061/978 0784413272.139>. Acesso em 20/02/2018.

VIELMO, A. L. Limite superior da retenção da água no solo: método de campo e método de estimativa. 2008. 81 f. Dissertação (Mestrado em Engenharia Agrícola), Universidade Federal de Santa Maria, Santa Maria.

VIONE, M. T. Modelagem matemática da evaporação da água do solo. 2007. 76 f. Dissertação (Mestrado em Modelagem Matemática), Departamento de Física, Estatística e Matemática da Universidade Regional do Noroeste do Estado do Rio Grande do Sul, Unijuí. 